\title{
Is vigorous physical activity important for (public) health?
}

\author{
Atividade física vigorosa é importante para a saúde (pública)?
}

\begin{tabular}{l} 
AUTHOR'S \\
\hline Michael Pratt $^{1}$ (D) \\
Victor Matsudo $^{2}$ (D \\
William E. Kraus ${ }^{3}$ iD \\
1 Herbert Wertheim School of Public Health \& \\
Human Longevity Science. University of California \\
San Diego, California, USA. \\
2 Centro de Estudos do Laboratório de Aptidão \\
Física de São Caetano do Sul - CELAFISCS, São \\
Caetano do Sul, São Paulo, Brasil. \\
3 Duke University School of Medicine in the \\
Division of Cardiology Medicine and the Duke \\
Molecular Physiology Institute. \\
CORRE SPONDING \\
Michael Pratt \\
mipratt@bealth.ucsd.edu \\
Herbert Wertheim School of Public Health \\
\& Human Longevity Science \\
University of California San Diego. 9500 \\
Gilman Dr. La Jolla, California, USA. \\
92093-0631.
\end{tabular}

$10.12820 /$ rbafs. $25 \mathrm{e} 0148$

\section{(cc) BY}

This work is licensed under a Creative Commons Attribution 4.0 International License.
Recommendations to the public on the quantity and quality of physical activity to improve health have long included vigorous physical activity. In fact, vigorous activity was the focus of most guidelines prior to the Centers for Disease Control and Prevention/American College of Sports Medicine recommendation published in $1995^{1}$. While much has changed in the ensuing 25 years - and even as public health recommendations have evolved to place a much greater emphasis on moderate physical activity ${ }^{2}-$ the general public and professionals remain intrigued by vigorous physical activity. This may in part be due to many researchers and clinicians having personal and professional backgrounds in sport and endurance exercise. In this commentary, we will look carefully at whether it is still justifiable for public health and clinical practitioners to retain a significant focus on vigorous physical activity. First, we will define "vigorous physical activity" and then attempt to address five questions about this issue:

1) Are there health benefits of vigorous intensity physical activity in addition to those of an equal volume of moderate intensity physical activity?

2) What are the risks associated with vigorous physical activity?

3) How much vigorous physical activity actually occurs at the population level?

4) Are there intervention strategies to increase and sustain vigorous physical activity in populations?

5) What are the health benefits of HIIT (High Intensity Interval Training)?

\section{Defining vigorous physical activity}

Vigorous physical activity may be defined in terms of absolute intensity (activity requiring 6.0 or greater METs or 7.0 or more $\mathrm{Kcal} /$ minute) or relative intensity (greater than $60 \%$ of maximal oxygen uptake or $70 \%$ of maximal heart rate). For public health purposes, the most commonly used definition is a threshold of greater than 6.0 METs coupled with examples such as walking very fast, running, or participating in an aerobics class ${ }^{2}$. However, because of age-associated decline in aerobic capacity (maximal oxygen uptake), some activities classified as moderate intensity in absolute terms (3.0 to less than 6.0 METS) may actually be vigorous for those over age 65 in relative terms ${ }^{2,3}$.

\section{Health benefits}

The added fitness benefits of vigorous physical activity over moderate physical activity are well established and obvious. Nobody would train to run a fast mile by simply walking for 30 minutes a day! However, the incre- 
mental benefits of an equal volume of vigorous physical activity compared with moderate physical activity are not easily identified and are outcome dependent. When controlling for amount, moderate intensity physical activity leads to improvements in insulin sensitivity, blood pressure, and body composition comparable to those resulting from vigorous intensity aerobic exercise ${ }^{4}$. There is some evidence of further reductions in all-cause mortality in U.S college alumni and Danes with sports participation and bicycle commuting after adjusting for leisure time activity ${ }^{5,6}$. However, these studies were not designed to answer the question of the incremental benefits of vigorous physical activity over moderate physical activity. They utilized self-report and thus could not clearly differentiate moderate from vigorous activity or control for total volume of physical activity. Very brief periods of presumptive vigorous intensity activity-such as stair climbing-clearly provide fitness benefits and may also provide health benefits ${ }^{2}$. In sum, the current scientific literature does not support the premise that vigorous physical activity provides consistent added benefits over an equal amount of moderate intensity physical activity.

\section{Health risks}

For almost all people and in almost all circumstances, the overall health benefits associated with increasing physical activity outweigh any added health risks ${ }^{7}$. However, vigorous physical activity in relative terms is associated with an increased risk of adverse cardiac events during the period of activity. Musculoskeletal injury risk increases with total volume of physical activity and with rapid increases in volume of activity, and may increase with intensity of physical activity; but, few studies have controlled for volume. Clinical and practical experience suggest greater injury risk with vigorous versus moderate intensity activity; however, these observations may be confounded by the greater activity volumes associated with vigorous physical activity ${ }^{7}$. Prolonged bouts of vigorous physical activity have been associated with transient reduction of immune function as measured by increased inflammatory biomarkers and risk of upper respiratory tract infections ${ }^{8}$. A consistent observation in many longitudinal studies of physical activity and all-cause mortality has been a slight uptick in mortality in persons with the very highest activity levels ${ }^{9}$. All-cause mortality for the most active persons (more than $10 \mathrm{x}$ recommended levels) is still lower than for sedentary persons but higher than that for persons who are active at recommended levels ${ }^{9}$. While it is again not possible to discern the composition of these large volumes of physical activity, it is much easier to reach very high volumes of physical activity if vigorous activity comprises a substantial portion of the total.

\section{Population levels of vigorous physical activity}

Until the advent of population-based accelerometry studies in the last decade, the tendency for over-reporting of both intensity and time of activity on questionnaires confounded assessments of population levels of vigorous physical activity. A recent study of physical activity levels assessed by accelerometry in 3865 subjects in eight cities in six countries found that adults averaged 20 minutes of vigorous physical activity per week with a range of 9 to 32 minutes ${ }^{10}$. In a representative urban sample of 630 adults in Cuernavaca, Mexico population-level vigorous physical activity was found to be only 6.7 minutes/week ${ }^{11}$. In an accelerometry-based sample of 414 adults in rural Malawi vigorous physical activity was remarkably low at less than $8 \mathrm{mi}^{-}$ nutes per week ${ }^{12}$. Based on the best available current evidence from studies in high, middle, and low-income countries using objective monitoring of physical activity, adults take part in an average of only 1 to $5 \mathrm{minu}-$ tes/day of vigorous physical activity.

\section{Interventions to increase vigorous physical activity}

Good surveillance is important for quantifying baseline levels of vigorous physical activity, but equally important for public health is being able to quantify intervention-induced changes in that low baseline. Unfortunately, very few community intervention studies have disaggregated vigorous from moderate intensity physical activity. Community-based physical activity classes are a promising intervention approach for increasing physical activity, probably including vigorous intensity activity ${ }^{13}$. For older adults it is possible that brisk walking might be a means by which to increase vigorous physical activity in relative terms. However, the proportion of vigorous versus moderate activity in community interventions and their effect sizes are currently unknown.

\section{And what about HIIT}

High Intensity Interval Training research has burgeoned in recent years-mostly in laboratory settings. It is a "rediscovery" of the classic principles of interval 
training first introduced by Woldemar Gerschler in the 1930s to train middle distance runners. HITT capitalizes on the high volume of activity associated with vigorous intensity and time efficiency to deliver predictably excellent fitness increases, improvements in insulin sensitivity, blood pressure, and body composition ${ }^{4}$. However, additional research is needed to address longer term adherence, population effect sizes, generalizability to more diverse populations, and injury risk across the wide variety of HIIT protocols ${ }^{14}$.

\section{Conclusions}

So, what do we know? Vigorous physical activity provides clear added fitness benefits and comparable health benefits to those from an equal volume of moderate intensity physical activity. Population levels of vigorous physical activity among adults are extremely low. Short term protocols such as HIIT can increase vigorous physical activity, improve fitness, and improve cardiometabolic risk factors. However, there is little to no evidence on the feasibility or sustainability of vigorous physical activity in community settings or at the population level. In order to close this knowledge gap, research is needed that can differentiate vigorous from moderate physical activity in diverse populations; over a wide age spectrum; and in a variety of community interventions. At present, given the very low baseline of population vigorous physical activity, no evidence on the feasibility of increasing vigorous activity in community populations, and no added health benefits of vigorous physical activity over equal volumes of moderate physical activity, we conclude that vigorous physical activity does not have an important place in public health. That said, it remains the right choice for a small subset of the population that may expand as better evidence accumulates.

\section{Conflict of interest}

The authors declare no conflict of interest.

\section{Author's contributions}

Dr. Pratt initially developed the concept for this paper. Drs. Pratt, Matsudo and Kraus discussed and further refined the concept. Dr. Pratt wrote the first draft and Drs. Pratt, Matsudo and Kraus reviewed, edited, contributed and approved all later drafts.

\section{References}

1. Pate RR, Pratt M, Blair SN, Haskell WL, et al. Physical activity and the public's health: a recommendation from the centers for disease control and prevention and the American College of Sports Medicine. JAMA, 1995;273:402-7.

2. 2018 Physical Activity Guidelines Advisory Committee. 2018 Physical Activity Guidelines Advisory Committee Scientific Report. Washington, DC: U.S. Department of Health and Human Services, 2018.

3. U.S. Department of Health \& Human Services. Physical Activity and Health: A Report of the Surgeon General. Atlanta, GA: U.S. Department of Health \& Human Services, Centers for Disease Control and Prevention, National Center for Chronic Disease Prevention and Health Promotion, 1996.

4. Campbell WW, Kraus WE, Powell KE, Haskell WL, Janza KF, Jakicic JM, et al. High-Intensity Interval Training for Cardiometabolic Disease Prevention. Med Sci Sports Exerc. 2019;51(6):1220-26.

5. Paffenbarger RS Jr, Hyde RT, Wing AL, Hsieh CC. Physical activity, all-cause mortality, and longevity of college alumni. N Engl J Med. 1986;314(10):605-13.

6. Andersen LB, Schnohr P, Schroll M, Hein HO. All-Cause Mortality Associated With Physical Activity During Leisure Time, Work, Sports and Cycling to Work. Archives of Internal Medicine. 2000;160(11):1621-28.

7. Physical Activity Guidelines Advisory Committee. Physical Activity Guidelines Advisory Committee Report, 2008. Washington, DC: U.S. Department of Health \& Human Services, 2008.

8. Nieman DC, LM Wentz. The compelling link between physical activity and the body's defense system. J Sport Health Sci 2019;8(3):201-17.

9. Arem H, Moore SC, Patel A, Hartge P, Gonzales AB, Visvanathan $\mathrm{K}$, et al. Leisure time physical activity and mortality: a detailed pooled analysis of the dose-response relationship. JAMA Internal Med. 2015;175(6): 959-67.

10. Cerin E, Cain KL, Oyeyemi AL, Owen N, Conway TL, Cochrane T, et al. Correlates of agreement between accelerometry and self-reported physical activity. Med Sci Sports Exerc. 2016;48(6):1075-84.

11. Salvo D, Torres C, Villa U, Rivera JA, Sarmiento OL, Reis $\mathrm{RS}$, et al. Accelerometer-based physical activity levels among Mexican adults and their relation with sociodemographic characteristics and BMI: a cross-sectional study. Int J Behav Nutr Phys Act. 2015;12(1):79.

12. Pratt M, Sallis JF, Cain KL, Conway TL, Palacios-Lopez A, Zezza A, et al. Physical activity and sedentary time in a rural adult population in Malawi compared with an agematched US urban population. BMJ Open Sport \& Exercise Medicine. 2020;0:e000812.

13. Heath GW, Parra DC, Sarmiento OL, Andersen LB, Owen $\mathrm{N}$, Goenka S, et al. Evidence-based intervention in physical activity: lessons from around the world. The Lancet 2012; 380(9838):272-81.

14. Stamatakis E, Johnson NA, Powell L, Hamer M, Rangul V, Holtermann A. Short and sporadic bouts in the 2018 US physical activity guidelines: is high-intensity incidental physical activity the new HIIT? Br J Sports Med. 2019.

Received: 08/10/2020

Approved: 19/10/2020 